\title{
Transport and acceleration of plasma in the magnetospheres of Earth and Jupiter and expectations for Saturn
}

\author{
M.G. Kivelson \\ Department of Earth and Space Sciences and Institute of Geophysics and Planetary Physics, University of California, Los Angeles, CA 90095-1567, USA
}

Received 8 September 2004; received in revised form 19 May 2005; accepted 19 May 2005

\begin{abstract}
The first comparative magnetospheres conference was held in Frascati, Italy thirty years ago this summer, less than half a year after the first spacecraft encounter with Jupiter's magnetosphere (Formisano, V. (Ed.), The Magnetospheres of the Earth and Jupiter, Proceedings of the Neil Brice Memorial Symposium held in Frascati, Italy, May 28-June 1, 1974. D. Reidel Publishing Co., Boston, USA, 1975). Disputes highlighted various issues still being investigated, such as how plasma transport at Jupiter deviates from the prototypical form of transport at Earth and the role of substorms in Jupiter's dynamics. Today there is a wealth of data on which to base the analysis, data gathered by seven missions that culminated with Galileo's 8-year orbital tour. We are still debating how magnetic flux is returned to the inner magnetosphere following its outward transport by iogenic plasma. We are still uncertain about the nature of sporadic dynamical disturbances at Jupiter and their relation to terrestrial substorms. At Saturn, the centrifugal stresses are not effective in distorting the magnetic field, so in some ways the magnetosphere appears Earthlike. Yet the presence of plasma sources in the close-in equatorial magnetosphere parallels conditions at Jupiter. This suggests that we need to study both Jupiter and Earth when thinking about what to anticipate from Cassini's exploration of Saturn's magnetosphere. This paper addresses issues relevant to plasma transport and acceleration in all three magnetospheres.
\end{abstract}

(C) 2005 Published by Elsevier Ltd on behalf of COSPAR.

Keywords: Magnetospheres; Outer planets; Jupiter; Saturn; Earth

\section{Three decades of comparing magnetospheres}

The first significant meeting on comparative magnetospheres (Formisano, 1975) took place in Frascati, Italy thirty years ago at the dawn of exploration of Jupiter's magnetosphere (half a year after the Pioneer 10 flyby). Subjects covered at the meeting included radio emissions, $\mathrm{X}$-ray emissions, transport, and, naturally, comparison with Earth's magnetosphere. Even Saturn was discussed by Scarf (1975), but facts were virtually lacking. For example, Scarf considered the possibility that Saturn would have no magnetic field, and speculated that the heliopause might lie inside of the 10 AU distance to Saturn's orbit. The importance of rotation relative to solar

\footnotetext{
E-mail address: mkivelson@igpp.ucla.edu
}

wind input for Jupiter, analyzed earlier by Brice and Ioannidis (1970) in a paper that is still cited today, was more fully discussed by Vasyliunas (1975). In other papers the issue of flow and transport was discussed a bit schizophrenically. Kennel and Coroniti (1975) asked if the observed field of Jupiter's middle and outer magnetosphere could arise from plasma outflow, as for a pulsar, but concluded that this wasn't likely. As we gather near the beginning of the third millennium, it is exciting to see how much we have learned and how much is left to understand.

\section{Overview and introduction to selected topics}

A review of particle acceleration and transport could, in principle, address a large number of topics. Table 1 
Table 1

Magnetospheric plasma

\begin{tabular}{ll}
\hline Sources and sinks & Transport and acceleration mechanisms \\
\hline $\begin{array}{l}\text { The solar wind }\left\{\begin{array}{l}\text { plasma } \\
\text { solar energetic particles }\end{array}\right. \\
\text { The ionosphere } \\
\begin{array}{l}\text { Rings } \\
\text { Cosmic rays }\end{array} \\
\text { CRAND } \\
\text { Moons }\left\{\begin{array}{l}\text { sputtered matter } \\
\text { outgassed matter }\end{array}\right. \\
\begin{array}{l}\text { Convection driven by the solar wind } \\
\text { Interchange } \\
\text { Reconnection }\end{array} \\
\text { Radial diffusion } \\
\text { Pitch angle scattering }\end{array}$ \\
Recirculation $\left\{\begin{array}{l}\text { through multiple charge exchange } \\
\text { through radial diffusion followed by } \\
\text { high latitude scattering }\end{array}\right.$ \\
\hline
\end{tabular}

provides a summary of particle sources and acceleration mechanisms that must be incorporated in a complete description of a planetary magnetosphere. The first column reveals the diversity of particle sources. The list includes external sources such as the solar wind and cosmic rays and internal sources such as the ionosphere and the surfaces and surrounding gases of rings and moons. The term cosmic ray albedo neutron decay (CRAND) refers to sources arising from a multi-step process in which cosmic rays impinging on matter within the magnetosphere liberate neutrons whose half life of $15 \mathrm{~min}$ is short enough that some of the free neutrons decay into protons while still within the magnetosphere. This process contributes to the plasma in Saturn's magnetosphere where the rings intercept cosmic rays effectively (see, for example, Paranicas et al., 1997).

The second column identifies some key mechanisms relevant to acceleration and transport. The term "convection" refers to motion of entire flux tubes under the combined influence of the solar wind and rotational stress imposed by the ionosphere. Strictly speaking the assumption that plasma remains on a flux tube as it moves through the magnetosphere can be applied only to the very low energy part of the distribution for which gradient-curvature drifts are negligible. For Earth this assumption is valid in the dipole field region for particles with energy less than $\mathrm{O}(1 \mathrm{keV})$ and for Jupiter the assumption is good to $\mathrm{MeV}$ energies. Higher energy particles drift significantly off the flux tube as they move through the inner magnetosphere.

Interchange, like convection, refers to motion of entire flux tubes but should strictly be limited to motions arising from a fluid instability driven by inertial effects like gravity and the centrifugal pseudo-force, as contrasted with convection in which the motion is imposed by hydromagnetic stresses. Interchange allows the motion of individual flux tubes with anomalous plasma content to differ from the motion of surrounding flux tubes. Because one may continue to assume that plasma is frozen to the flux tube, the relation $\mathbf{E}=-\mathbf{v} \times \mathbf{B}$ remains valid in the MHD approximation, so small scale electric field reversals must be present where the flow reverses sense locally. Because interchange and convection both relate to the motion of flux tubes as a whole, convection can be considered a form of interchange with a different dominant driver.

Reconnection is an important mechanism for both acceleration and transport. At Earth, it is critical to substorm-related injections of energetic particles and, in most cases, seems to be driven externally. At Jupiter, there is evidence for reconnection and associated acceleration, but no consensus on whether it is driven primarily by the solar wind (Khurana, 2001) or by the instability of the internal plasma (Kivelson and Southwood, 2005).

Additional transport/acceleration mechanisms may be both energy and mass dependent. They include radial and pitch angle diffusion, charge exchange, and various processes involving spatial recirculation of ions. Diffusion refers to the spreading of a distribution of plasma particles either in velocity or configuration space through the action of randomly fluctuating fields. Diffusion may be particularly effective for a particular energy/ unit charge as when particle drift periods match the periods of the fluctuations.

Charge exchange refers to a process in which a neutral particle moving at velocity $v_{\mathrm{n}}$ exchanges a charge with an ion moving at velocity $v_{\mathrm{i}}$, thereby modifying the energy distribution in the plasma and providing a source of neutrals moving at the speed of the local plasma. Charge exchange at Earth serves as a loss process for ring current ions. At Jupiter it serves as an important transport mechanism because the newly created neutrals originating in the Io plasma torus can be flung out to hundreds of jovian radii before being re-ionized (Mendillo et al., 1990). Neutrals liberated from the rings and moons of Saturn also participate in charge exchange. In all three systems, the neutrals serve as tracers of their plasma sources, so valuable images have been generated by creative data-processing of the directional measurements of their fluxes (Roelof, 1987).

Recirculation provides a mechanism for accelerating ions to high energies. One idea, still not confirmed by observations, was first explored by Nishida (1976) who observed that solar wind ions (energy of a few eV) could be adiabatically accelerated to $>1 \mathrm{keV}$ by inward diffu- 
sion at Jupiter, but that adiabatic acceleration could not account for the presence of $>\mathrm{MeV}$ ions throughout much of the magnetosphere. He suggested that if diffusing ions reaching the inner magnetosphere were subsequently scattered non-adiabatically outward across Lshells and allowed to diffuse back in again, they could be adiabatically accelerated to $\mathrm{MeV}$ energies. The nonadiabatic transport required for returning ions from low L-shells to high L-shells could be achieved by invoking fluctuating fields at the ionospheric ends of the innermost field lines. A somewhat different multi-step process was discussed for heavy ion acceleration by Barbosa et al. (1984). They remarked that the process of charge exchange near Io's orbit produces heavy neutrals that are flung outward on ballistic trajectories. Such neutrals can undergo a second charge exchange in Jupiter's outer magnetosphere, where the high azimuthal flow speed would enable the ions to pick up some hundreds of $\mathrm{keV}$ energy. Inward adiabatic diffusion could then accelerate them to $\mathrm{MeV}$ energies.

The diversity of plasma populations and of transport and acceleration mechanisms is merely touched on above. Limitations of space require selectivity, so this paper focuses on one subject that is important in all planetary magnetospheres and a second subject that is relevant to understanding Jupiter's magnetosphere. The more general topic addresses observational constraints on models of the mechanisms that accelerate particles (specifically electrons) to $>\mathrm{MeV}$ (relativistic) energies. Details are provided by studies carried out at Earth, which is probably the best laboratory for learning about this matter, but one can speculate on how the results found at Earth apply to other planetary magnetospheres. For Jupiter, this paper addresses the challenging question of how plasma introduced at Io moves through the system and how it is lost. A brief summary identifies ways in which the processes identified at Earth and Jupiter are likely to apply to Saturn.

\section{Acceleration of electrons to relativistic energy}

Intermittent, significant increases of relativistic electron flux in the terrestrial magnetosphere are known to produce adverse phenomena that affect many systems of societal importance, including spacecraft, communications and power grids. During a geomagnetic storm, electron fluxes increase by orders of magnitude on time scales of $\sim 1$ day. The flux of $>\mathrm{MeV}$ electrons increases as close to Earth as $L=3-4$. Here $L$ is a parameter that characterizes the distance in Earth radii $\left(R_{\mathrm{E}}\right)$ to the equatorial portion of a flux tube.

It is not yet clearly established how the acceleration is accomplished. Drift in the dawn-dusk direction parallel or antiparallel to the large scale magnetospheric electric field can accelerate particles. Acceleration in this manner leads typically to energies of order $100 \mathrm{keV}$. The inductive electric field associated with substorms can produce higher particle energies and in large substorms can account for $\mathrm{MeV}$ electrons injected into the nightside magnetosphere beyond geostationary orbit at $6.6 R_{\mathrm{E}}$, but the inductive field does not significantly accelerate particles in the regions closer to Earth. A separate mechanism is needed to explain the electron peak near $L \sim 3-4$ that develops in $\sim 1$ day following a geomagnetic storm.

Various acceleration mechanisms have been proposed; they fall into two distinct classes. Some describe how a source population in the outer magnetosphere beyond $L=6.6$ can be driven inward and further accelerated by inward diffusion. The diffusion is driven by ultralow frequency (ULF, less than the ion gyrofrequency) fluctuations of electric and magnetic fields. Other proposed mechanisms describe ways in which a pre-existing particle population at low $L$ can be accelerated in situ by very low frequency (VLF, but greater than the ion gyrofrequency) waves.

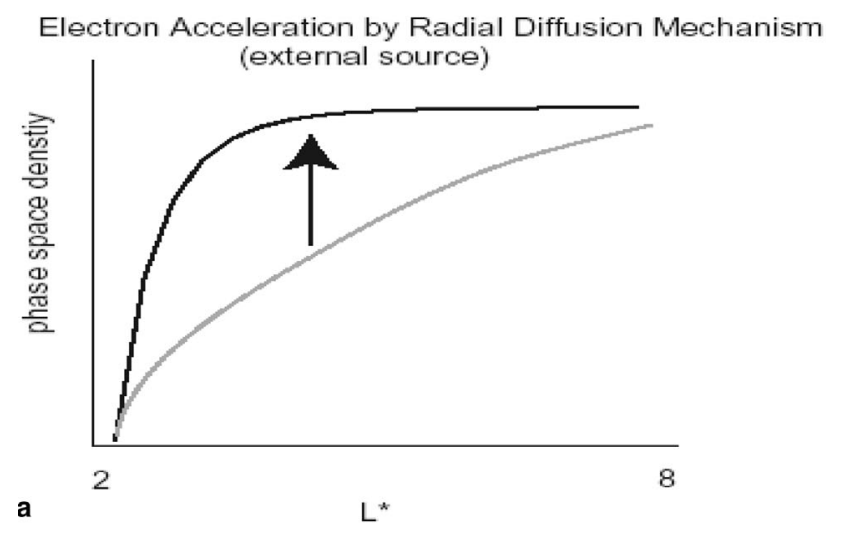

Electron Acceleration by Internal Source Mechansim

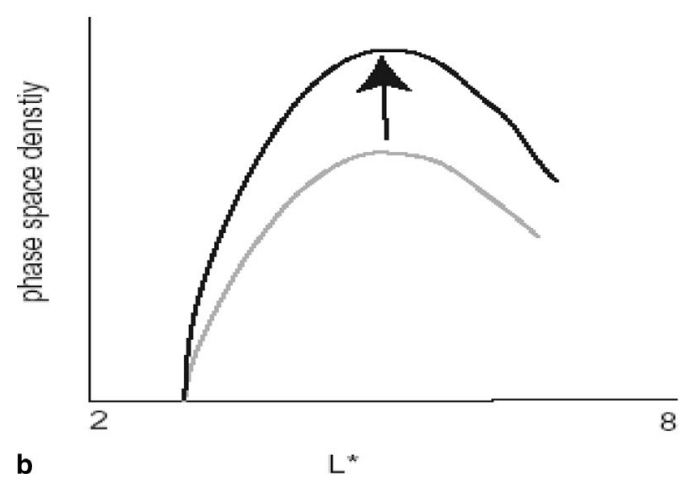

Fig. 1. Schematic forms of phase space density vs. radial distance expressed in terms of $L^{*}$ produced by different acceleration mechanisms. In both cases the gray curves show an initial state and the black curves a later state. (a) Distribution arising from inward diffusion from a source at $L^{*}>8$. (b) Distribution arising from acceleration by a process acting at $L^{*}<8$. 
The two classes of acceleration mechanisms can be distinguished experimentally by determining how the phase space density expressed in terms of canonical variables (Schulz and Lanzerotti, 1974) varies with $L$ and time. Diffusion can cause the phase space density on other L shells to increase, but only up to the level in the source region. The limitation is implicit in the Liouville equation that governs the phase space density as a function of canonical coordinates such as the adiabatic invariants commonly used in magnetospheric physics (Northrop, 1963; Kivelson, 1995). Thus if the acceleration is linked to diffusion inward from the outer magne- tosphere, the phase space density must have a nonnegative radial gradient throughout the inner magnetosphere (Fig. 1(a)). On the other hand, acceleration in situ can produce a negative outward gradient (Fig. 1(b)).

It may seem perplexing that, after years of data analysis, we do not know which class of model is applicable. The primary difficulty is that spacecraft instruments measure the local magnetic field $\mathbf{B}$ and the particle flux at given energy $(W)$, pitch angle $(\alpha)$ and position $(\mathbf{R})$. Conversion from flux to phase space density is straightforward, but conversion of the variables from those measured to canonical variables such as $\mu, j$ or $K$, and

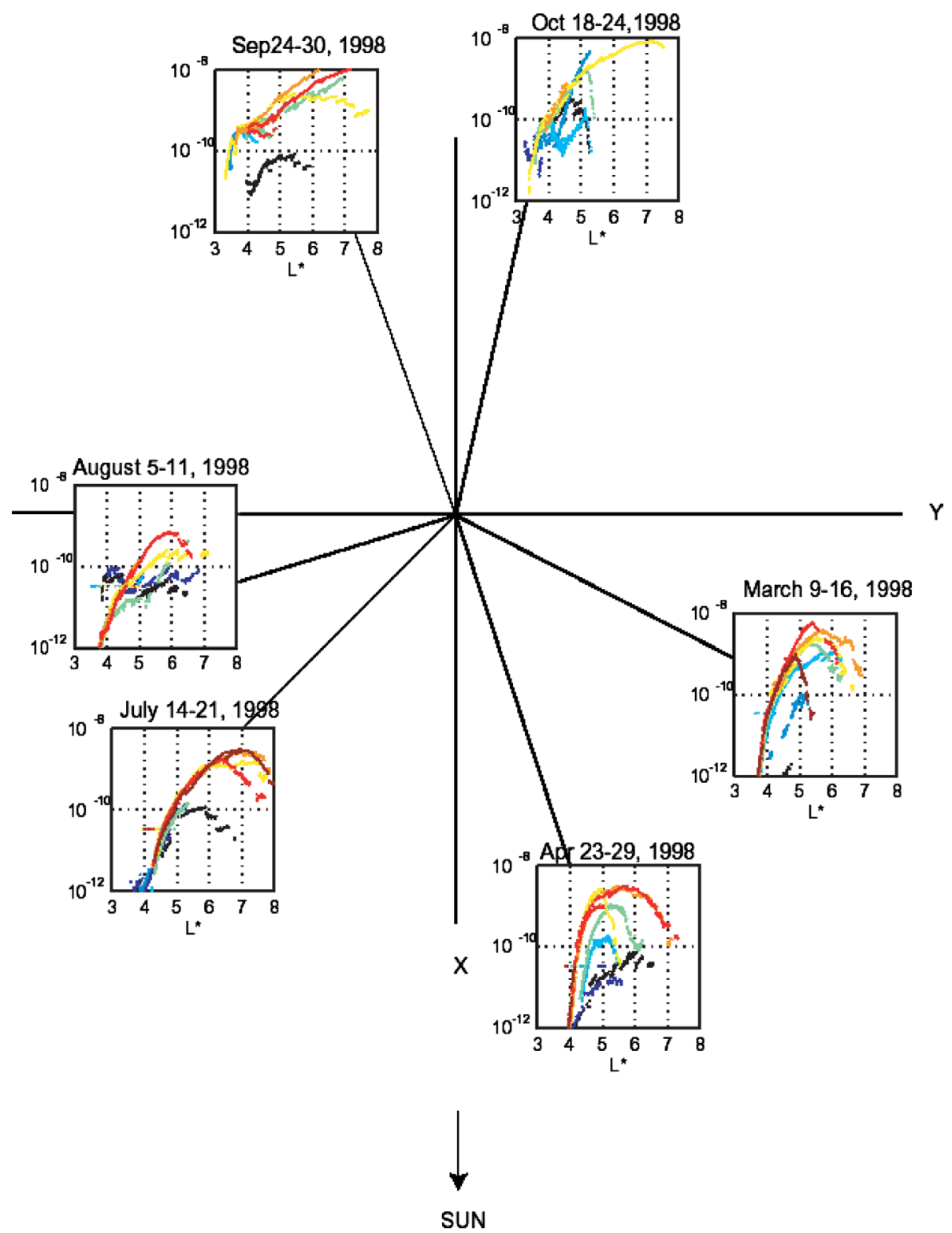

Fig. 2. Adapted from Green and Kivelson (2004). Phase space density of relativistic electrons as a function of $L^{*}$ during storm periods. The data were based on measurement by the CEPPAD investigation on the POLAR spacecraft but are transformed into invariant coordinates using a magnetic field model. The inserts are organized by local time with noon at the bottom and midnight at the top. Dayside profiles are seen to peak near $L^{*}=5$. Nightside profiles are very disorganized because they are sensitive to details of the magnetospheric magnetic field model that are poorly constrained. 
$L^{*}$ (Roederer, 1970) is not. The first adiabatic invariant, $\mu=W_{\perp} / B$, involves only local values (where $W_{\perp}$ is the particle's thermal energy perpendicular to the local magnetic field direction) and thus presents no problem. The other adiabatic invariants require knowledge of the properties of the magnetic field over a large spatial volume in addition to the properties of the particles and the field at the spacecraft location. $K$ is roughly proportional to the length of the field line, a quantity that is very uncertain particularly if measurements are made substantially off the equator and $L^{*}$ provides an invariant measure of the distance from the center of the Earth to the equatorial crossing of the flux tube. Green and Kivelson (2004) use the Tsyganenko (Tsyganenko, 1995; Tsyganenko and Stern, 1996) field model to convert fluxes of relativistic electrons measured by the Polar CEPPAD (Blake et al., 1995) investigation into phase space density in the required coordinate system. Fig. 2 provides examples of the distribution functions versus $L^{*}$ at selected local times. For each local time the plots show several profiles measured on successive passes separated by approximately $18 \mathrm{~h}$.

A striking feature of the plots is the fact that the radial profiles of the four dayside panels peak at relatively low $L^{*}$ and then decrease whereas the radial profiles of the nightside passes are variable and most increase monotonically with $L^{*}$. The drift periods for the energetic electrons being investigated is of order $10 \mathrm{~min}$, so it is extremely unlikely that their radial profiles can differ so greatly and so systematically as a function of local time if they have been properly expressed in terms of canonical variables. Thus the day-night differences are likely to arise because of deficiencies of the field model during active times. Green and Kivelson test this speculation by allowing the $D s t$ parameter in the Tsyganenko field model to change from its nominal value $D s t_{\mathrm{o}}$ to another value: $D s t^{\prime}=D s t_{\mathrm{o}} \pm 40 \mathrm{nT}$. They find that the form of dayside profiles is qualitatively robust for different field models but the nightside profiles vary markedly in form as different models are used, sometimes changing from monotonic to non-monotonic functions of $L^{*}$. Thus they select the dayside profiles with peaks at low $L^{*}$ for further study.

Peaks at low $L^{*}$ can arise not only through local acceleration but also through inward radial diffusion followed by losses at large $L^{*}$. However, the temporal sequence of radial profiles plotted in Fig. 3 shows that the peaks arise at low $L^{*}$ without a source at larger
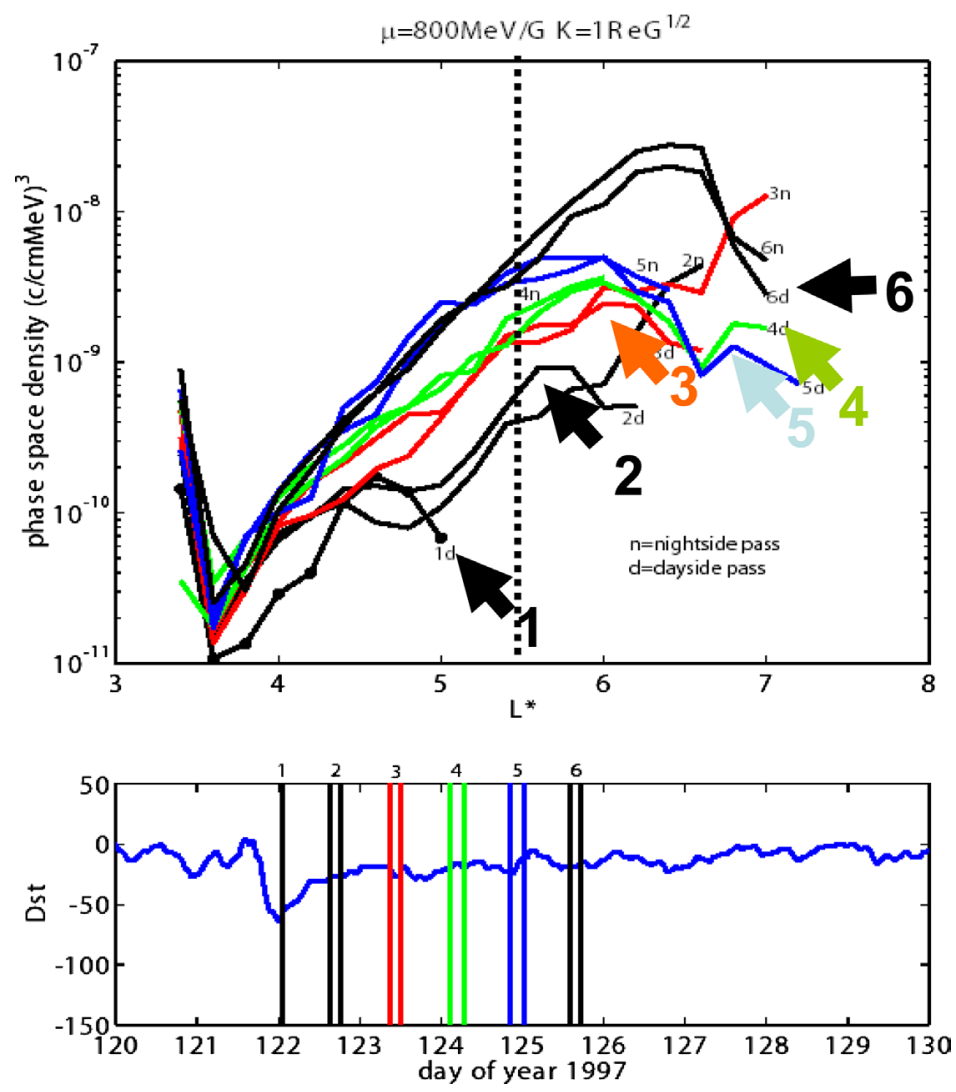

Fig. 3. Adapted from Green and Kivelson (2004). Variation of the profiles of phase space density vs. $L^{*}$ on successive Polar passes. POLAR's polar orbit takes it through both the day and night sides of the magnetosphere on each pass. The lower panel shows the Dst variation during the geomagnetic storm interval. Color coding show when the profiles were obtained. The forms of the dayside profiles, which are insensitive to details of the magnetic field model, show that the peak at low $L^{*}$ grows without a source of energetic electrons at large $L^{*}$. 

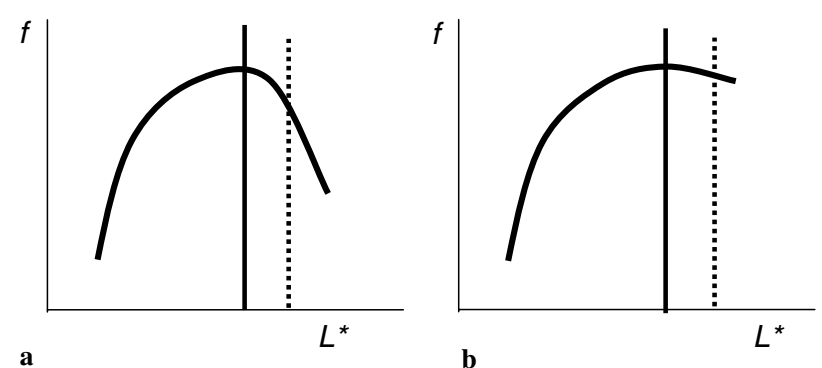

Fig. 4. Schematic profiles of phase space density of relativistic electrons vs. $L^{*}$. In (a) the profile peaks at low $L^{*}$ typical of in situ acceleration. In (b), representing a later time, radial diffusion has spread the distribution away from the peak. The dashed line represents $L^{*}$ for geostationary orbit and the flux at that position is higher at the later time (b) than initially (a).

$L^{*}$. This allows Green and Kivelson to conclude that acceleration occurs in situ at low $L^{*}$, most probably through a mechanism involving scattering by VLF waves.

Many studies (Liu et al., 1999; Mathie and Mann, 2000; Green and Kivelson, 2001; Elkington et al., 2003) have demonstrated a strong correlation between ULF wave power and relativistic electron fluxes at geostationary orbit. As noted earlier, this is often taken as evidence that the ULF waves are responsible for the acceleration. However, if the above argument is correct and acceleration occurs at low $L^{*}$ through an in situ mechanism, ULF waves will cause distribution to diffuse both inward and outward from the peak at low $L^{*}$. Thus the association of ULF waves and flux enhancements at geostationary orbit may result from a two step process illustrated in Fig. 4, with initial acceleration occurring inside of $L=6.6$ and the appearance of relativistic electrons at geostationary orbit resulting from outward diffusion mediated by the ULF waves. Indeed there are reports (Mathie and Mann, 2000) that flux enhancements at geostationary orbit require persistent ULF power for a prolonged time after the main phase of a storm, a result that is easily explained by the two step model. In a recent paper O'Brien et al. (2003) argue that both VLF and ULF waves are important for the acceleration of relativistic electrons.

In situ acceleration by VLF waves is likely not to be the only mechanism for acceleration to relativistic energies, but it seems important at Earth. We have less evidence of the mechanisms through which electrons and ions are accelerated at Jupiter and Saturn. At Earth, dramatic acceleration referred to as injection occurs during geomagnetic storms. There is no clear analogue of such storms at Jupiter and Saturn, yet one cannot rule out the possibility that analogous acceleration processes are maintaining the steady level of energetic electrons that is found in these distant magnetospheres. On the
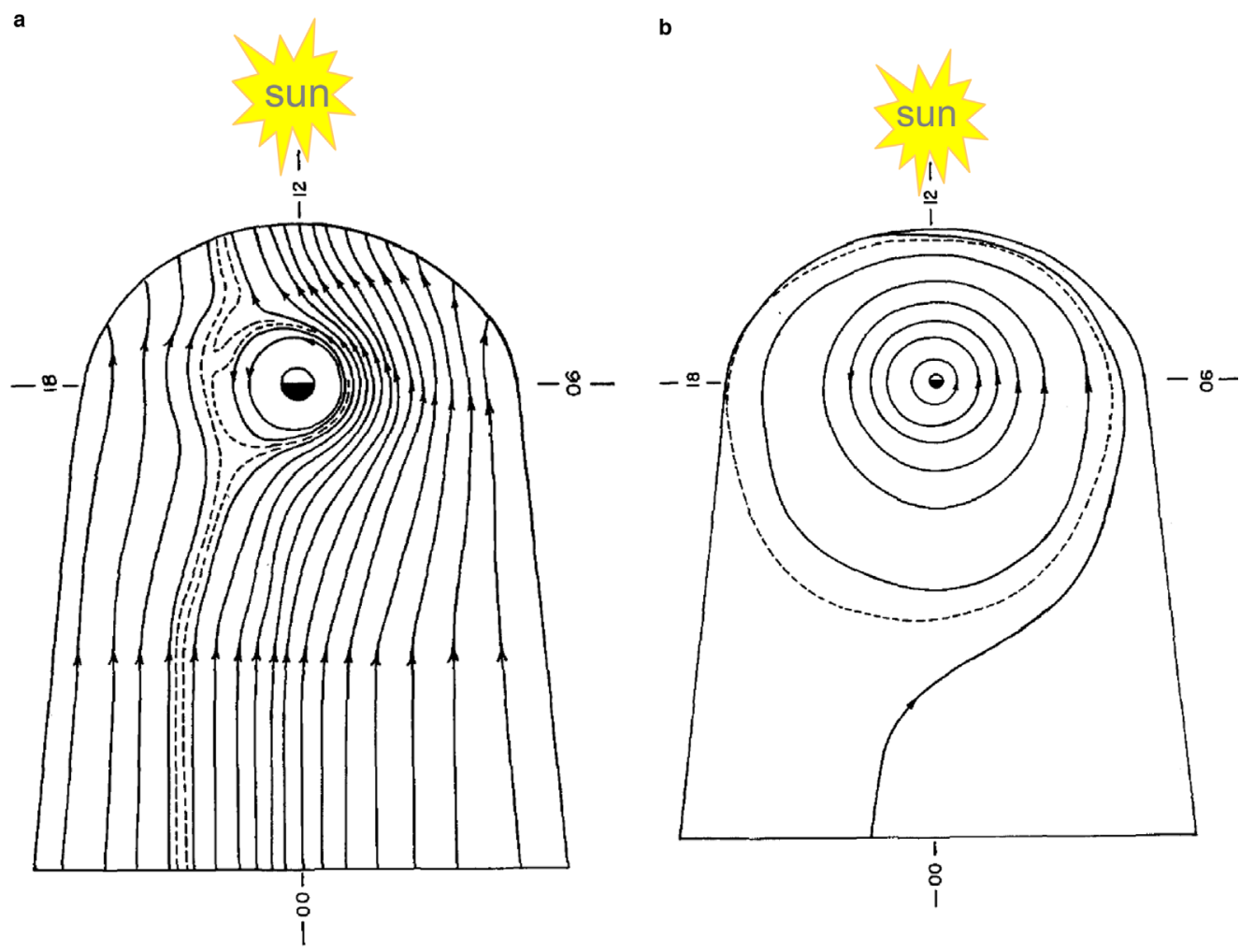

Fig. 5. Adapted from Brice and Ioannidis (1970). Flow streamlines for plasma at Earth (above) and Jupiter (below). Dashed curves are separatrices for the flow. 
other hand, the important acceleration mechanisms may be very different. For example, the possible role of recirculation has been noted. Identification of the relative contributions of various acceleration mechanisms acting in outer planet magnetospheres is a challenging and important problem that possibly could be studied with data already acquired for Jupiter or to be acquired soon for Saturn.

\section{Transport of iogenic plasma at Jupiter}

Let us next turn to Jupiter, focusing on how the plasma introduced deep in the magnetosphere at the orbit of Io is lost. The issue is central to understanding the functioning of the magnetosphere and to characterizing the ways in which Jupiter's magnetosphere differs from Earth's. Excellent background for this discussion can be found in the new book on Jupiter (Bagenal et al., 2004). Outward transport and loss of the plasma introduced near Io's orbit at a rate of 1 ton/s (Hill, 1979) is required for an approximately steady state magnetosphere. The energy of the source plasma is relatively low, with typical ion energy of $\sim 100 \mathrm{eV}$ and electron energy of 5-100 eV (Kivelson et al., 2004). For such low energy plasma, radial diffusion is slow. Convective outflow can remove the plasma but decreases magnetic flux, a conserved quantity that must return in some manner. Brice and Ioannidis (1970) pointed out that whereas at Earth only the inner magnetosphere is rotation-dominated, at Jupiter the entire magnetosphere is. The patterns (see Fig. 5) imply that at Earth, flux tubes crossing the dawn meridian beyond roughly $0.4 R_{\mathrm{mp}}$ and the dusk meridian beyond $\sim 0.6 R_{\mathrm{mp}}$, where $R_{\mathrm{mp}}$ is the distance to the subsolar magnetopause, flow into the dayside boundary. Following reconnection of the Sunward moving flux tubes, magnetic flux returns to the night side of the terrestrial magnetosphere over the poles as described by Dungey (1961). However, at Jupiter only the very outermost flux tubes on the dawn side beyond say $0.9 R_{\mathrm{mp}}$ can reach the magnetopause, participate in dayside reconnection and return flux over the poles. (On the dusk side, a model including a uniform cross tail field and full corotation puts the stagnation point near $6 R_{\mathrm{E}}$ for Earth but well outside the dusk magnetopause for Jupiter. It must also be outside the

Interchange event inbound to Io

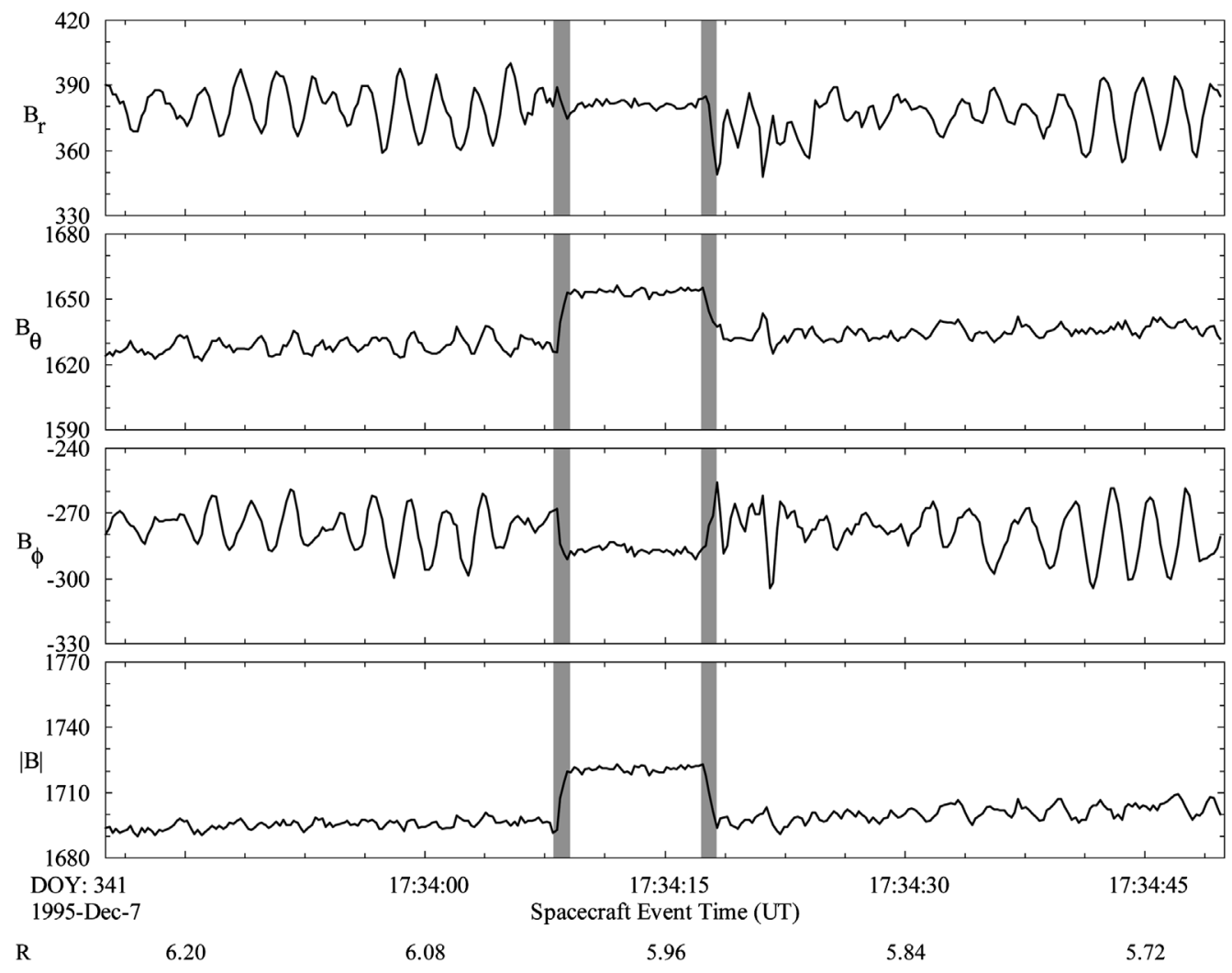

Fig. 6. Galileo magnetometer measurements of three components of the magnetic field and its magnitude on December 7, 1996 just outside the orbit of Io (radial distance from Jupiter in $R_{\mathrm{J}}$ is labeled below the universal time of the measurements). The fluctuations in $B_{\mathrm{r}}$ and $B_{\phi}$ are ion cyclotron waves arising from newly picked up $\mathrm{SO}_{2}+$ ions. The vertical bars identify a short-duration increase of the field magnitude. During the field increase the waves disappear but they return shortly after. 
magnetopause for Saturn. In neither case are Sunward flow paths present in the dusk hemisphere.) This means that at Earth it is possible for all of the plasma returning from neutral lines in the tail to convect around an equatorial exclusion zone and return to the dayside magnetopause. At Jupiter and Saturn, very little of the plasma present on the night side within $\sim 150 R_{\mathrm{J}}$ flows from the night side to the day side. The dilemma, then, is to understand how the iogenic plasma moves outward through the extensive region of rotating plasma while conserving magnetic flux.

One mechanism that can achieve the required objective is the interchange instability. This plasma instability arises if the system is not in its lowest free energy state. For example, an atmosphere develops an adiabatic temperature profile vs. altitude because for higher lapse rates, any small displacement of a parcel of air results in anomalous buoyancy for that parcel (motion downward under gravity if anomalously dense, motion upward if anomalously tenuous), so it continues to move until it reaches equilibrium. At Jupiter outside of $\sim 2$ $R_{\mathrm{J}}$, the "buoyancy" effect is dominated by the centrifugal pseudo-force, so the parcels with higher flux tube content move out, not in. As they move out, they are replaced by other parcels with lower flux tube content moving in.

A limited number of observations in support of the interchange picture are available, with the clearest cases documented in the near neighborhood of Io. A square wave increase of the magnetic field magnitude bounded by abrupt steps is unusual in a region well displaced from the location of a moon, but a particularly clear example of this type of signature occurred on Galileo's first pass by Io (Kivelson et al., 1997). Fig. 6 shows a portion of the magnetometer data. During the $10 \mathrm{~s}$ interval of importance, which occurred shortly before the Io encounter but well away from Io, the field magnitude increased by $\sim 1 \%$ and the large amplitude ion cyclotron waves present in the background plasma disappeared but returned when the field returned to the background level. Fig. 7 replots the trace of the field magnitude and shows the simultaneously measured plasma wave data (Bolton et al., 1997) and energetic particle flux (Thorne et al., 1997). The thermal energy of torus ions is $\sim 100$ $\mathrm{eV}$; assuming pressure balance, a $1 \%$ change in field magnitude requires a change of plasma density of order $2300 \mathrm{~cm}^{-3}$. The plasma density in this region of the torus is $3-4000 \mathrm{~cm}^{-3}$ (Frank et al., 1996) so this change is significant. A drop of plasma density is confirmed by the sharp decrease of the frequency of upper hybrid waves at the time of the field increase. The flux of energetic particles also increases abruptly. Other examples of this type of event near Io are discussed by Kivelson et al. (1997) and Crary et al. (1998) and additional possible cases have been reported in the inner magnetosphere by Russell et al. $(2000,2001)$.

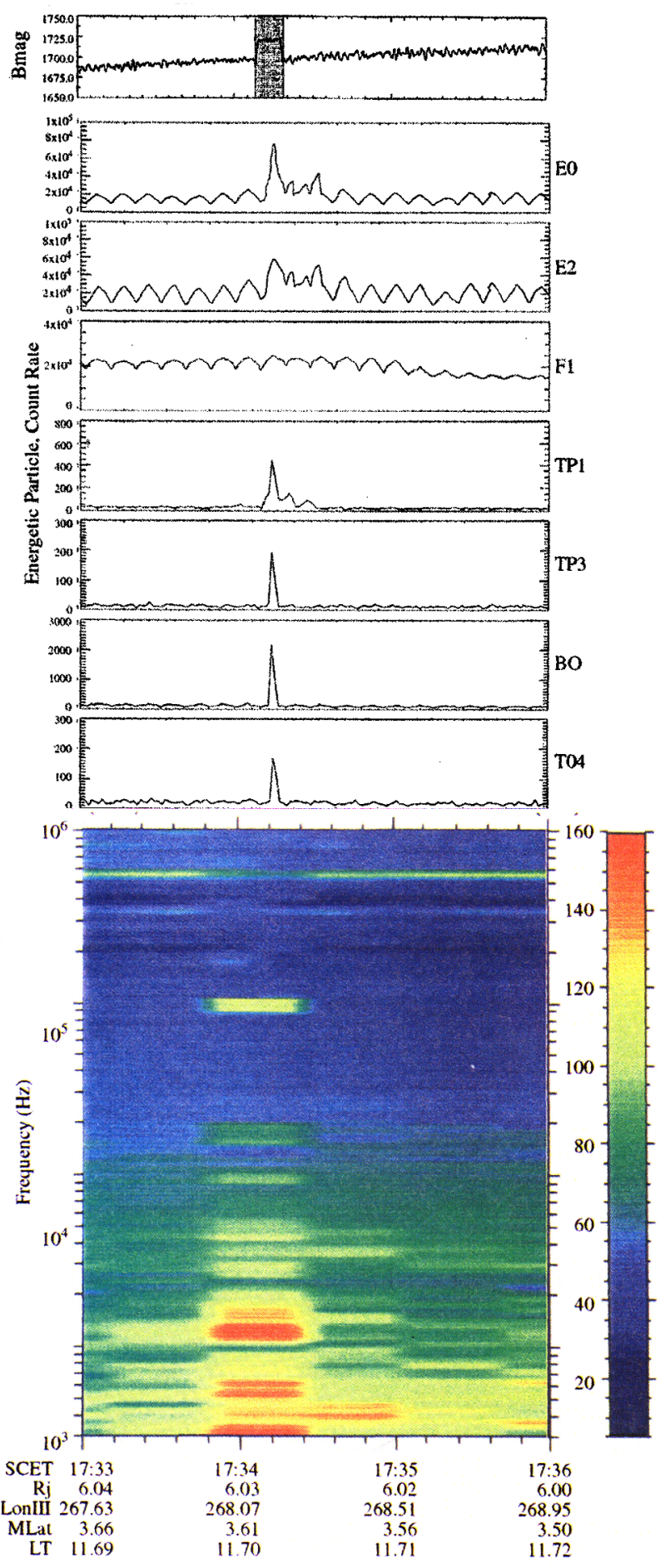

Fig. 7. Adapted from Thorne et al., 1997. In order from the top, $B_{\text {mag, }}$ the magnetic field magnitude as in the lower panel of Fig. 6, E0, E2, $\mathrm{F} 1$, energetic electron fluxes in different energy channels, TP1, TP3, $\mathrm{BO}, \mathrm{T} 04$, energetic ion fluxes in different energy channels, frequencytime dynamic spectrum of the VLF wave power showing a drop in frequency of the intense yellow band at the upper hybrid resonance frequency.

The data are consistent with short duration encounters with flux tubes containing plasma that differs from the background plasma by having a lower thermal plasma 
density and a higher energetic particle flux. The flux tubes have been interpreted as being tubes of small cross section (of order an ion gyroradius) in rapid inward motion and hence observed in Galileo data for times from a few seconds to about $1 \mathrm{~min}$. Such flux tubes can be identified only with the full time resolution Galileo data $(\leqslant 0.33 \mathrm{~s} /$ vector for the magnetometer), which, because of the failure of the high gain antenna, were available only for brief intervals mainly during flybys of the Galilean moons. For this reason, good statistics are lacking and most of the observations are found in data from the first flyby of Io.

In the magnetotail one might expect to observe interchange as square wave depressions of the field produced when overdense flux tubes drift outward; the scale of the outward-moving flux tubes may be sufficiently large that they can be detected in medium resolution ( $\sim 24 \mathrm{~s} /$ vector $)$ data. Increases and drops of limited duration few minutes and abrupt onset and termination are found. An example from Galileo magnetometer data acquired near $37 R_{\mathrm{J}}$ at about 0500 LT is shown in Fig. 8. The decreases in $|B|$ could result from plasma sheet flapping but it seems improbable for flapping to be sufficiently impulsive to produce the abrupt decreases and relatively flat minima that would arise from an interchanging flux tube. It is difficult to test the speculation because total pressure should be constant in either case and both cases would be characterized by having antiphase relations between changes of the plasma density and the field magnitude.

Despite the paucity of evidence, it is plausible to think that interchange solves the plasma transport prob- lem. Most probably magnetic flux is carried out with the slowly outflowing plasma in the overdense flux tubes and returned through rapid inflow of small transversescale, under-dense flux tubes.

One may wonder whether the mechanism of outward transport of iogenic plasma can be identified through computer simulations. Unfortunately, the Jovian magnetosphere is so large that MHD models are required to study global scale processes, and inevitably such simulations cannot represent processes on the small scales proposed for interchange. Yet the conservation of magnetic flux is fundamental to well structured computer simulations, so the models are forced to return flux to the inner magnetosphere in some way. In a simulation by Walker et al. (2001), a vortex forms in the post-dusk sector, with inflow returning flux at least to some inner boundary (Fig. 9). Flow data from Galileo energetic particle analysis (Krupp et al., 2001) does show duskside slowing but, although there are a few inward-pointing vectors in the data of Krupp et al. (2001), the inferred velocity vectors do not support a significant vortical flow pattern. The Io plasma transport problem remains imperfectly understood and calls for more work, especially more correlative studies of field and plasma properties.

\section{Loss of iogenic plasma from the outer magnetosphere}

The above section considered how, in a quasi-steady manner, the iogenic plasma moves out through the inner

\section{Ganymede 7 (s3rh)}

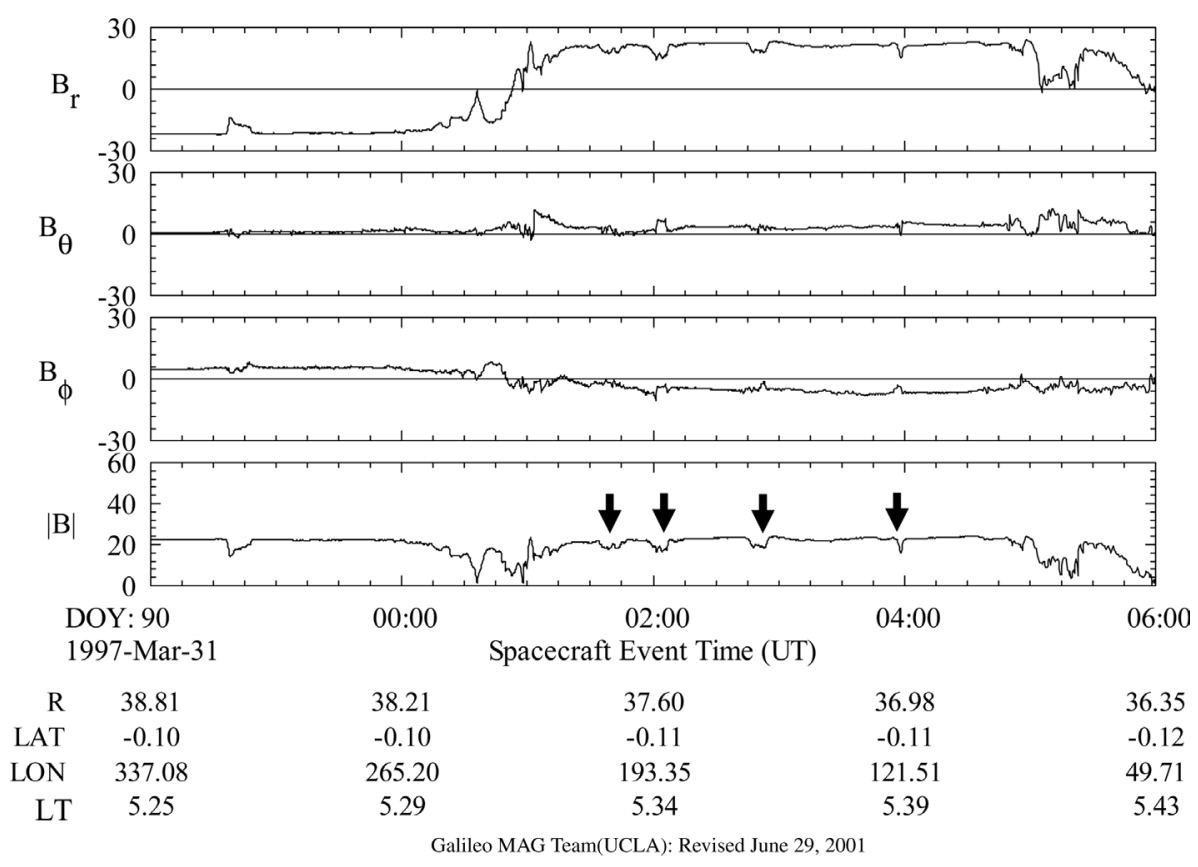

Fig. 8. Galileo magnetometer measurements of three components of the magnetic field and its magnitude on March 31, 1997. Of interest are the dips in the field magnitude that are quite abrupt and of short duration marked by arrows in the lowest panel. 


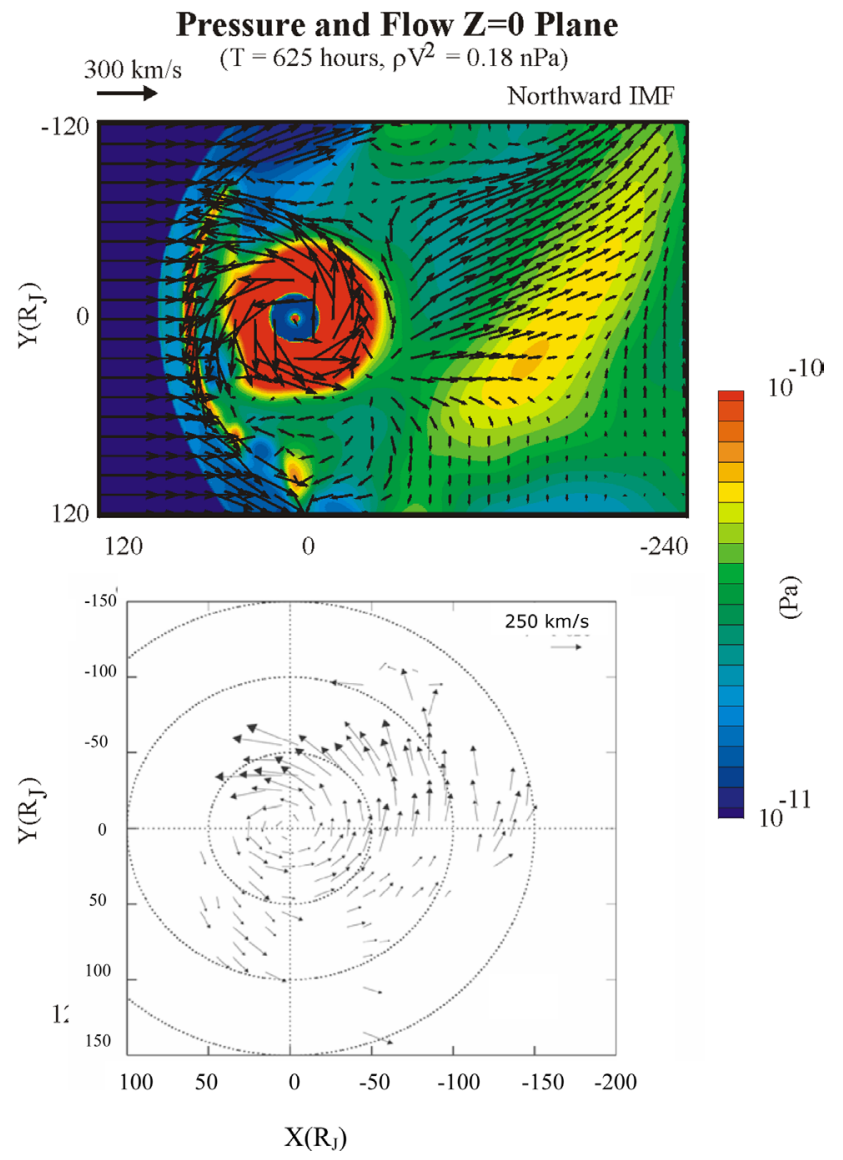

Fig. 9. Above: From Walker et al. (2001), the equatorial plane of a simulation of the Jovian magnetosphere. Color represents pressure and the arrows represent flows. Below: Flow vectors vs. LT in the Jovian equatorial regions inferred from anisotropy of energetic particle fluxes by Krupp et al. (2001).

and middle magnetosphere of Jupiter. Ultimately, the plasma must leave the system through the outer magnetosphere, but this process is also not readily explained. At Earth, plasma can readily convect to the dayside magnetopause and reconnect with the solar wind or can reconnect in the magnetotail, get trapped in a plasmoid and sweep down the tail. What happens at Jupiter?

Signatures with the magnetic (Russell et al., 1998) and particle (Woch et al., 1999) characteristics of reconnection have been identified in Jupiter's magnetotail. Fig. 10 shows the magnetic field measured on June 18, 1997 between 0200 and 0300 local time near $71 R_{\mathrm{J}}$ on Galileo's eighth orbit close to the neutral sheet where the radial component $\left(B_{\mathrm{r}}\right)$ reverses sign. The reversal of sign of the north-south component (positive $B_{\theta}$ points southward, which is the quiet time orientation of the Jovian magnetic field) is the signature expected as a flux rope or a plasmoid flows past the spacecraft. Woch et al. (1999) use anisotropy of energetic particle fluxes to characterize flows. They find that rapid Sunward and tailward flows are often present in the night- time sector across a separatrix that cuts across the tail near $100 R_{\mathrm{J}}$ and shifts further downtail as it crosses from pre-midnight to post-midnight. The fastest flows are found in the post-midnight sector and this has been interpreted in terms of a solar wind-controlled convection cell principally affecting flows in the post midnight region by Khurana (2001) and Walker et al. (2001). At Earth field and flow signatures of the sort described here would typically arise during a substorm and would be followed by major reconfiguration of the tail, but no dipolarization follows in this or other similar events observed at Jupiter. The question then is how should we understand nightside reconnection at Jupiter?

The spatial extent of the solar wind driven convection cell is not firmly established but the arguments of Section 4 on the dominant role of rotation in the Jovian magnetosphere make it unlikely that reconnection with open flux tubes in the lobes of the magnetotail dominate the tail dynamics. Reconnection signatures are indubitably present, but they do not require that flux tubes of the plasmasheet are reconnecting with open flux tubes as they do in a terrestrial substorm. Reconnection can also occur through the stretching and "breaking" of closed flux tubes. The process was illustrated by Vasyliunas (1983) in a much reproduced diagram (Fig. 11). Flux tubes filled with iogenic plasma are carried around the dayside just inside the magnetopause that confines them. As they rotate around past dusk, the confining force of the magnetopause no longer acts against the centrifugal stresses to limit outward motion of equatorial plasma. The equatorial plasma moves down the tail, stretching the flux tube. Stretching is followed by plasma depletion close to the planet and an X-line forms producing a plasmoid. Bubbles of plasma go off down the tail.

Does anyone worry about how a plasmoid on a closed flux tube gets away? It is a bit tricky. One may wonder if the outward-moving plasmoid can be blocked from downtail motion by the confining effect of lobe field lines that are reconnecting at a distant neutral line further down the tail. If that is the case, as at Earth, the plasmoid is free to move down tail only after lobe field lines have reconnected at the inner edge of the plasmoid; their curvature force can then propel the newly detached plasmoid down the tail. At Jupiter, the solar wind is not a primary source of convection, so if portions of the plasmasheet plasma break off and head down tail at high speed, they are likely to have sufficient momentum to push the lobe flux tubes aside and flow out down the tail. Furthermore, the plasmoid can move diagonally across the tail following the directions of some of the fast flows reported by Krupp et al. (1998) and reach the magnetopause on the morning-side flank. With a finite azimuthal component of the flow, there is likely to be some loss through the morningside flanks.

The plasmoid breaking picture is not inconsistent with the pattern of high speed near-equatorial flows dis- 


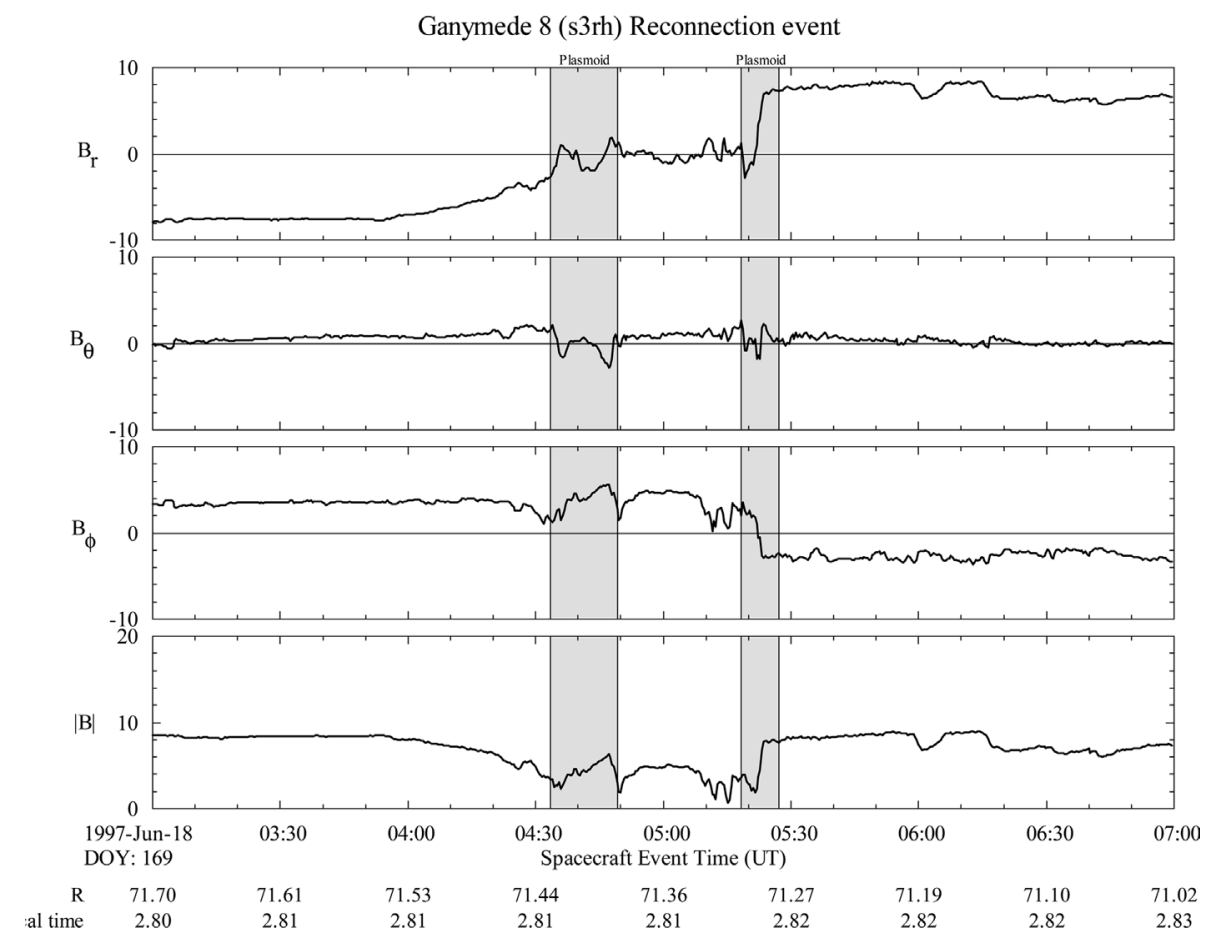

Galileo MAG Team(UCLA): Revised June 29, 2001

Fig. 10. As for Fig. 8 but for June 18, 1997. Radial distance in $R_{\mathrm{J}}$ and local time are indicated below the universal time axis. Galileo in the magnetotail observed repeated reversals of the southward field component near the crossing of the tail current sheet. The signatures are thought to be those of plasmoids.

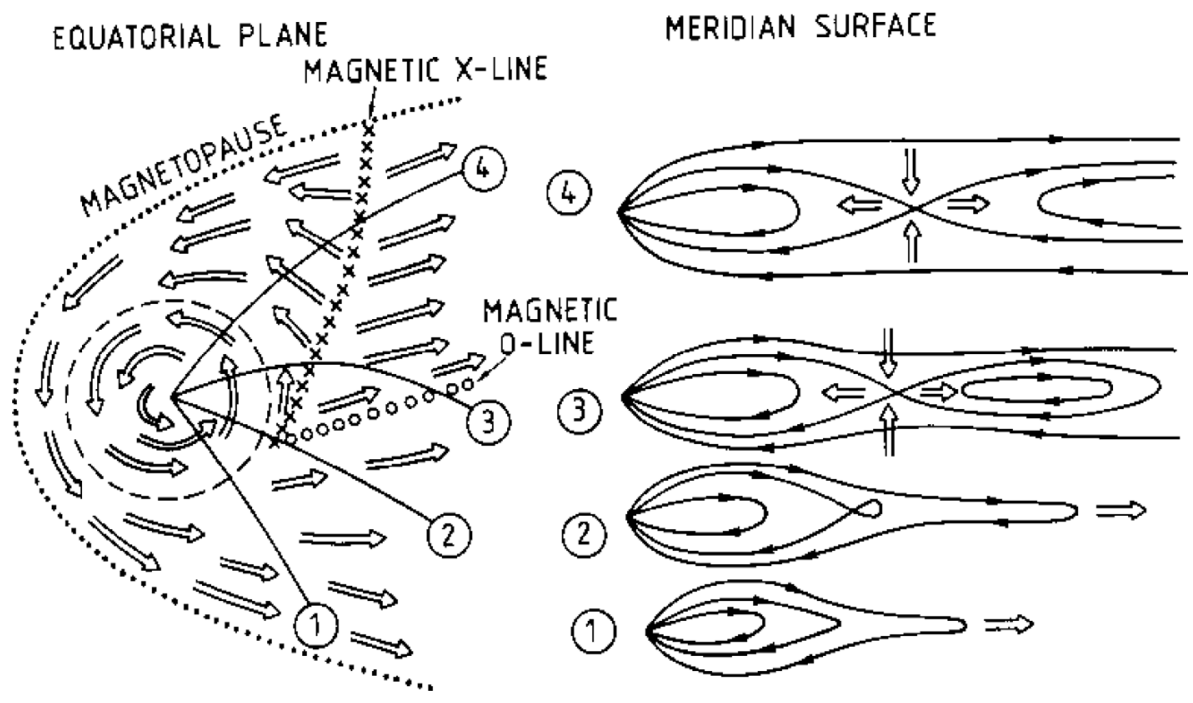

Fig. 11. From Vasyliunas (1983). A schematic of the flow pattern expected in the equatorial plane of the Jovian magnetosphere on the left. Schematics of the field and flow patterns expected in orthogonal cuts made at locations indicated by numbers in the equatorial plane are shown in the meridian surface the right.

cussed in connection with possible substorm-like behavior. Stretching initiates as a flux tube rotates past dusk into a region where it can no longer be confined by the magnetopause. The stretching increases as the flux tube rotates towards midnight, and it is not unreason- able to think (as in the Vasyliunas picture) that breaking occurs at the part of the distended flux tube near midnight from which the plasma has been lost. Following the breaking, the portion of the flux tube linked to Jupiter will have lost much of its plasma load, and the 
inward curvature force will no longer be balanced by outward plasma and energetic particle pressures. The flux tube will try to snap inward, driving flows in the region when they are observed, although its motion will be impeded by the plasma closer to the planet. The bubble that has broken off will produce the field reversal signatures illustrated in Fig. 10 as it moves down the tail.

In summary, then, the dynamics of the outer portion of the Jovian magnetosphere can generate plasma and field signatures that are analogous in some ways to those observed at Earth during substorms, but it is likely that at Jupiter the dynamics are principally driven by rotational stresses and that lobe field reconnection occurs primarily in a spatially confined region near the dawn flanks and is not significant for the energy budget of Jupiter's magnetosphere.

\section{Lessons for Saturn}

Saturn is in many ways intermediate between Jupiter and Earth in its dynamical properties. Like Jupiter, it is rotation-dominated, so it is reasonable to look for evidence of interchange as an important transport mechanism. Saturn's plasma density is generally lower than is Jupiter's, especially in the inner magnetosphere, which implies that inertial stresses linked to rotation are less significant. The dayside field, for example, is only slightly distorted by rotation. Breaking off of plasmoids in the tail seems unlikely because the inertial stresses should be much less important than at Jupiter. Of particular importance is the diversity of particle sources and the presence of rings containing solid matter that serves as a source or a sink of charged particles. The early reports indicate that these features produce exciting dynamics particularly in the energetic neutral imaging data.

Because Saturn is much like Earth, significant solar wind-driven responses are likely. It is not clear that dayside reconnection will lead to substorms, but it seems quite likely that it will. There are some reports of pulsating dayside auroral features at Jupiter (Gladstone et al., 2002), of small scale transient Earth-like reconnection signatures at Jupiter (Grodent, 2004; Grodent et al., 2004), and of extreme auroral variability at Saturn (Grodent, 2004). Some features of the variability could plausibly be related to reconnection of lobe flux tubes. If substorms are significant, there could also be storm intervals driven by unusually intense dayside reconnection. If so, it would be likely that fluxes of relativistic electrons would increase; it is possible that the acceleration is caused by VLF waves as seems true at Earth. Prognostication is dangerous, especially when the truth will be known so soon. The first reports on Saturn from the Cassini team confirm that significant scientific advances will follow from the exploration that is now underway.

\section{Acknowledgements}

This work was supported in part by the Atmospheric Sciences Division of the National Science Foundation under grant NSF ATM 02-05958 and by NASA under contract JPL 1238965. The author acknowledges with gratitude the contributions of Janet Green (now at LASP, University of Colorado) whose dissertation forms the basis of the discussion of electron acceleration, and of her colleagues Raymond J. Walker, Krishan K. Khurana, and David J. Southwood whose lively discussions helped her develop the prejudices displayed in this paper.

\section{References}

Bagenal, F., Dowling, T., McKinnon, W. Jupiter: The Planet, Satellites and Magnetosphere. Cambridge University Press, New York, 2004.

Barbosa, D.D., Eviatar, A., Siscoe, G.L. On the acceleration of energetic ions in Jupiter's magnetosphere. J. Geophys. Res 89, 3789, 1984.

Blake, J.26 co-authors CEPPAD: comprehensive energetic particle and pitch angle distribution experiment on polar. Space Sci. Rev. 71, 531, 1995.

Bolton, S.J., Thorne, R.M., Gurnett, D.A., Kurth, W.S., Williams, D.J. Enhanced whistler-mode emissions: signatures of interchange motion in the Io torus. Geophys. Res. Lett. 24, 2123-2126, 1997.

Brice, N.M., Ioannidis, G.A. The magnetospheres of Jupiter and Earth. Icarus 13, 173, 1970.

Crary, F.J., Bagenal, F., Frank, L.A., Paterson, W.R. A region of disturbed plasma within the Io torus: Galileo Plasma Spectrometer Observations, in: AGU 1998 Fall Meeting, SM21A-11, 1998.

Dungey, J.W. Interplanetary magnetic field and the auroral zones. Phys. Rev. Lett. 6, 47-48, 1961.

Elkington, S.R., Hudson, M.K., Chan, A.A. Resonant acceleration and diffusion of outer zone electrons in an asymmetric geomagnetic field. J. Geophys. Res. 108 (A3), 1116, doi:10.1029/2001JA009202, 2003.

Formisano, V. (Ed.). The Magnetospheres of the Earth and Jupiter. Proceedings of the Neil Brice Memorial Symposium held in Frascati, Italy, May 28-June 1, 1974. D. Reidel Publishing Co., Boston, USA, 1975.

Frank, L.A., Paterson, W.R., Ackerson, K.L., Vasyliunas, V.M., Coroniti, F.V., Bolton, S.J. Plasma observations at Io with the Galileo spacecraft. Science 274, 394-395, 1996.

Gladstone, G.R. et al. A pulsating auroral X-ray hot spot on Jupiter. Nature 415 (28 February), 1000, 2002.

Green, J.C., Kivelson, M.G. A tale of two theories: How the adiabatic response and ULF waves affect relativistic electrons. J. Geophys. Res. 106, 25,777, 2001.

Green, J.C., Kivelson, M.G. Relativistic electrons in the outer radiation belt: Differentiating between acceleration mechanisms. J. Geophys. Res. 109, A03213, doi:10.1029/2003JA010153, 2004.

Grodent, D. Auroral processes at Earth, Jupiter and Saturn, in: Eos Trans. AGU 85(17), Joint Assembly Suppl., Abstract SM12A-04, 2004.

Grodent, D., Gerard, J.-C., Clarke, J.T., Gladstone, G.R., Waite Jr., J.H. A possible auroral signature of a magnetotail reconnection process on Jupiter. J. Geophys. Res. 109, A05201, 10.1029/ 2003JA010341, 2004.

Hill, T.W. Inertial limit on corotation. J. Geophys. Res. 84, 6554-6558, 1979. 
Kennel, C.F., Coroniti, F.V. Is Jupiter's magnetosphere like a pulsar's or Earth's?. in: Formisano, V. (Ed.), The Magnetospheres of the Earth and Jupiter. D. Reidel Publishing Co, Boston, USA, p. 451, 1975.

Khurana, K.K. The influence of solar wind on Jupiter's magnetosphere deduced from currents in the equatorial plane. J. Geophys. Res. 106, 25,999, 2001.

Kivelson, M.G. Physics of space plasmas. in: Kivelson, M.G., Russell, C.T. (Eds.), Introduction to Space Physics. Cambridge University Press, New York, pp. 27-57, 1995.

Kivelson, M.G., Southwood, D.J. Dynamical consequences of two modes of centrifugal instability in Jupiter's outer magnetosphere, J. Geophys. Res, in revision (UCLA Institute of Geophysics and Planetary Physics Publication \# 6219), 2005.

Kivelson, M.G., Khurana, K.K., Russell, C.T., Walker, R.J. Intermittent short-duration plasma-field anomalies in the Io plasma torus: Evidence for interchange in the Io plasma torus?. Geophys. Res. Lett. 24, 2127, 1997.

Kivelson, M.G., Bagenal, F., Kurth, W.S., Neubauer, F.M., Paranicas, C., Saur, J. Magnetospheric interactions with satellites. in: Bagenal, F., Dowling, T., McKinnon, W. (Eds.), Jupiter: The Planet, Satellites and Magnetosphere. Cambridge University Press, New York, 2004.

Krupp, N., Woch, J., Lagg, A., Wilken, B., Livi, S., Williams, D.J. Energetic particle bursts in the predawn Jovian magnetotail. Geophys. Res. Lett. 25, 1249-1252, 1998.

Krupp, N., Woch, J., Lagg, A., Roelof, E.C., Williams, D.J., Livi, S., Wilken, B. Local time asymmetry of energetic ion anisotropies in the Jovian magnetosphere. Planet. Space Sci. 49, 283-289, 2001.

Liu, W.W., Rostocker, G., Baker, D.N. Internal acceleration of relativistic electrons by large-amplitude ULF pulsations. J. Geophys. Res. 104, 17,391, 1999.

Mathie, R.A., Mann, I.R. A correlation between extended intervals of ULF wave power and storm-time geosynchronous relativistic electron flux enhancements. Geophys. Res. Lett. 27, 3261, 2000.

Mendillo, M., Baumgardner, J., Flynn, F., Hughes, W.J. The extended sodium nebula of Jupiter. Nature 348, 312-314, 1990.

Nishida, A. Outward diffusion of energetic particles from the Jovian radiation belt. J. Geophys. Res 81, 1771, 1976.

Northrop, T.G. The Adiabatic Motion of Charged Particles. Interscience, New York, 1963.

O’Brien, T.P., Lorentzen, K.R., Mann, I.R., Meredith, N.P., Blake, J.B., Fennell, J.F., Looper, M.D., Milling, D.K., Anderson, R.R. Energization of relativistic electrons in the presence of ULF power and $\mathrm{MeV}$ microbursts: Evidence for dual ULF and VLF acceleration. J. Geophys. Res. 108, 1329, doi:10.1029/2002JA009784, 2003.
Paranicas, C., Cheng, A.F., Mauk, B.H., Keath, E.P., Krimigis, S.M. Evidence of a source of energetic ions at Saturn. J. Geophys. Res. 102, 17459-17466, 1997.

Roederer, J.G. Dynamics of Geomagnetically Trapped Radiation. Springer-Verlag, New York, 1970.

Roelof, E.C. Energetic neutral atom image of a storm-time ring current. Geophys. Res. Lett. 14, 652, 1987.

Russell, C.T., Khurana, K.K., Huddleston, D.E., Kivelson, M.G. Localized reconnection in the near Jovian magnetotail. Science 280 (15 May), 1061, 1998.

Russell, C.T., Kivelson, M.G., Kurth, W.S., Gurnett, D.A. Implications of depleted flux tubes in the Jovian magnetosphere. Geophys. Res. Lett. 27, 3133, 2000.

Russell, C.T., Kivelson, M.G., Kurth, W.S., Gurnett, D.A. Depleted magnetic flux tubes as probes of the Io torus plasma. Adv. Space Res. 28 (10), 1489, 2001.

Scarf, F. The magnetospheres of Jupiter and Saturn. in: The Magnetospheres of the Earth and JupiterProceedings of the Neil Brice Memorial Symposium held in Frascati, Italy, May 28-June 1, 1974. D. Reidel Publishing Co., Boston, USA, 1975.

Schulz, M., Lanzerotti, L.J. Particle Diffusion in the Radiation Belts. Springer-Verlag, New York, 1974.

Thorne, R.M., Williams, D.J., McEntire, R.W., Armstrong, T.P., Stone, S., Bolton, S., Gurnett, D.A., Kivelson, M.G. Galileo evidence for rapid interchange transport in the Io torus. Geophys. Res. Lett. 24, 2131, 1997.

Tsyganenko, N.A. Modeling the Earth's magnetospheric magnetic field confined within a realistic magnetopause. J. Geophys. Res 100, 5599, 1995.

Tsyganenko, N.A., Stern, D.P. Modeling the global magnetic field of the large-scale Birkeland current systems. J. Geophys. Res 101, 27187, 1996.

Vasyliunas, V.M. Concepts of magnetospheric convection. in: Formisano, V. (Ed.), The Magnetospheres of the Earth and Jupiter. D. Reidel Publishing Co., Boston, USA, p. 179, 1975.

Vasyliunas, V.M. Plasma distribution and flow. in: Dessler, A.J. (Ed.), Physics of the Jovian Magnetosphere. Cambridge University Press, New York, pp. 395-453, 1983.

Walker, R.J., Ogino, T., Kivelson, M.G. Magnetohydrodynamic simulations of the effects of the solar wind on the Jovian magnetosphere. Planet. Space Sci. 49, 237, 2001.

Woch, J., Krupp, N., Khurana, K.K., Kivelson, M.G., Roux, A., Perraut, S., Louarn, P., Lagg, A., Williams, D.J., Livi, S., Wilken, B. Plasma sheet dynamics in the Jovian magnetotail: Signatures for substormlike processes?. Geophys. Res. Lett. 26, 2137-2140, 1999. 